\title{
The effect of intestinal bile on the stability of lipid-based vesicular system used as oral drug carriers
}

\author{
Mosab Arafat* \\ Mosab Arafat, Assistant Professor, College of Pharmacy, Al Ain University of Science and Technology, Al Ain, PO Box 64141, Al Ain, Abu Dhabi, UAE
}

\begin{abstract}
Many lipid-based vesicles such as liposomes have been investigated as a colloidal carrier for oral drug delivery systems. However, their low stability in the gastrointestinal tract is still a major issue. A solubilisation of the phospholipids membrane triggered by intestinal bile salts in the small intestine causes premature release of the bioactive compound prior to intestinal absorption. In contrast, inclusion of bile salt in the preparation of lipid-based vesicular systems seems to play an important role in stability by enhancing the vesicles integrity upon exposure to intestinal bile salt in the gut. Thus, the present review gives a suggestion on further understanding of colloidal and interfacial mechanisms underlying the role of bile salt in the partitioning, transport and uptake processes as this is might be a key toward optimum strategies improving the stability of lipid-based vesicles.
\end{abstract}

Intestinal bile salts are bio-surfactants existing in the gastrointestinal tract (GIT) which have essential role either on fat and lipid digestion or absorption [1]. Acidic lipases, which are secreted by the gastric chief cells in the fundic mucosa of the stomach, regulate $10-30 \%$ of the lipid hydrolysis. In the other hand, pancreatic lipases make up to $90 \%$ of lipid hydrolysis in the small intestine. The process is supported by bile salts distributed through a network of bile ducts, first secreted from hepatocytes, transported viabile canaliculi, exit the liver via hepatic duct, joined with cystic and pancreatic duct to finally enter the small intestine. This hepatic bile contains large quantities of bile acids, cholesterol and other organic molecules. The main function of bile salt is to enhance emulsification and absorption of oils and lipoids upon aggregations. Thus, intestinal bile salts have an immediate effect on the lipolysis process of lipid-based components [2]. Similarly, lipidbased colloidal systems used for oral drug delivery system such as liposomes are expected to be subjected to these digestion processes. Being highly susceptible to the detergent effect of intestinal bile salt they tend to undergo destruction of the phospholipids bilayer and ultimately the solubilisation of the vesicular membrane [3]. As a result of the solubilising effect, a premature leakage of entrapped bioactive compounds might occur in the gut prior to intestinal absorption.

The lipolysis can take place on the surface as well as the interface of the lipid vesicles. Herein, the demolishing effect on the bilayer is dependent on the high bile salt concentration of the environment. A decreased integrity can convert the vesicles into mixed micelles based on the interaction of bile salt with lipids, causing further leakage to the entrapped bioactive compounds [4-8]. Exposed to the intestinal environment would be an issue for many drug, being either hydrolytically degradation by acidity or enzymes, or they might crush out due to low solubility.

The lipid composition of vesicles has been shown to influence the stability against intestinal bile salts and enzymes [9]. Furthermore, the incorporation of a particular amount of exogenous bile salt into lipid-based vesicles can resist the disruption and degradation through intestinal bile salts [10]. Thereby, inclusion of bile salts as an integral part in the vesicle formulation can stabilize lipid-bilayer membranes against the further detergent effects of intestinal bile salts through repulsion [11]. The amount and type of exogenous bile salt to be incorporated in the lipid-based vesicles is critical and it mainly depends on theircritical micelles concentration (CMC) [11]. Table 1 shows different types of bile salts, their CMC and their aggregation constant related to their hydrophobicity [12].

The amphipathic nature of exogenous bile salts is essential on enhancing the absorption of bioactive compounds by increasing their solubility and altering the membrane permeability by interacting with the local phospholipids [13]. Thus, bile salt can be used as penetration enhancers in drug formulations to support mucosal absorption especially due to the membrane destabilizing properties $[14,15]$. It was shown that bile salts can significantly enhance the intestinal permeability and oral bioavailability of class III drug [16,17]. Consequently, the incorporation of bile salt has a double positive effect protecting the vesicular integrity and supporting the transport through the intestinal mucosa.

Even being successful, only a few examples have been shown in literature. Almost 50 years ago, bile salts were described to enhanced transport of poorly absorbable drugs through the intestinal mucosa [18]. Mixed micelles of lipids and bile salts were shown to increase oral bioavailability of poorly water-soluble silybin [19], Cucurbitacin B [20] or benzodiazepines [21], as well as acid-labile cefotaxime [16]. However, the bilosomes research has been mainly focused on oral vaccine delivery $[10,22,23]$ or the protection of other peptidomimetic compounds such as insulin [24]. Lately, the application has been extended to hydrophobic drugs, for example fenofibrate [25], or the

Correspondence to: Mosab Arafat, Assistant Professor, College of Pharmacy, $\mathrm{Al}$ Ain University of Science and Technology, Al Ain, PO Box 64141, Al Ain, Abu Dhabi, UAE, E-mail: Mosab.arafat@aau.ac.ae

Received: December 02, 2016; Accepted: December 21, 2016; Published: December 23, 2016 
Table 1. Overview of critical micelle concentration (CMC), aggregation number and aggregation constant for different bile salts.

\begin{tabular}{|c|c|c|c|}
\hline Bile salt types & Minimum CMC & Maximum CMC & Aggregation constant \\
\hline Deoxycholate & $2 \mathrm{mM}$ & $5 \mathrm{mM}$ & 17 \\
\hline Chenodeoxycholate & $6 \mathrm{mM}$ & $9 \mathrm{mM}$ & 15 \\
\hline Ursodeoxycholate & $2 \mathrm{mM}$ & $19 \mathrm{mM}$ & 5 \\
\hline Cholate & $4 \mathrm{mM}$ & $20 \mathrm{mM}$ & 7 \\
\hline
\end{tabular}

acid-labile cefotaxime [17].

Today, lipids-based vesicular drug delivery systems are progressively being utilized for delivering various bioactive compounds. Hence, the integration of exogenous bile salt in the lipid bilayer membrane can support the development and preparation of stable vesicular systems to protect a wide range of sensitive active compounds. The use of bile salts combines two of their major advantageous properties which are as fellow: 1) acting as permeation enhancer agent and 2) physically stabilizing the lipid-based vesicles to prevent premature release of the bioactive compounds, and finally degradation the gut before mucosal uptake. In this regards, considering the bile salt incorporation in the formulation of lipid-based vesicular systems is beneficial. However, a further understanding of the colloidal and interfacial mechanisms underlying the role of bile in the partitioning, transport and uptake processes is a key to lead to optimum strategies on improving the stability of lipid-based vesicles in oral administration.

\section{References}

1. Parsegian VA, Rand RP, Rau DC (2000) Osmotic stress, crowding, preferential hydration, and binding: A comparison of perspectives. Proc Natl Acad Sci U S A 97: 3987-3992. [Crossref]
2. Ayrapetyan SN, Suleymanyan MA, Saghyan AA, Dadalyan SS (1984) Autoregulation of the electrogenic sodium pump. Cell Mol Neurobiol 4: 367-383. [Crossref]

3. Ayrapetyan SN, Arvanov VL, Maginyan SB, Azatyan KV (1985) Further study of the correlation between Na-pump activity and membrane chemosensitivity. Cell Mol Neurobiol 5: 231-243. [Crossref]

4. Ayrapetyan SN, Rychkov GY, Suleymanyan MA (1988) Effects of water flow on transmembrane ionic currents in neurons of Helix pomatia and in squid giant axons. Comp Biochem Physiol A Comp Physiol 89: 179-186. [Crossref]

5. Ayrapetyan SN (2016) Cell hydration-induced changes of membrane conductivity as a marker for estimation of biological effects of chemical and physical factors on organism. J Bioequiv Availab 8:10000e72.

6. Lehninger AL (1970) Mitochondria and calcium ion transport. Biochem J 119: 129138. [Crossref]

7. Baker PF, Blaustein MP, Hodgkin AL, Steinhardt RA (1969) The influence of calcium on sodium efflux in squid axons. J Physiol 200: 431-458. [Crossref]

8. Blaustein MP, Lederer WJ (1999) Sodium/calcium exchange: its physiological implications. Physiol Rev 79: 763-854. [Crossref]

9. Xie Z, Askari A (2002) $\mathrm{Na}(+) / \mathrm{K}(+)$-ATPase as a signal transducer. Eur J Biochem 269 2434-2439. [Crossref]

10. Saghian AA, Ayrapetyan SN, Carpenter DO (1996) Low concentrations of ouabain stimulate $\mathrm{Na} / \mathrm{Ca}$ exchange in neurons. Cell Mol Neurobiol 16: 489-498. [Crossref]

11. Narinyan L, De J, Ayrapetyan S (2014) Age-dependenti in $\mathrm{Ca}^{2+}$ exchange magnetosensitivity in rat heart muscles. Biochemistry and Biophysics 2: 1-3

12. Brini M, Carafoli E (2009) Calcium pumps in health and disease. Physiol Rev 89: 13411378. [Crossref]

13. Heqimyan A, Narinyan L, Nikoghosyan A, Ayrapetyan S (2015) Age-dependent magnetic sensitivity of brain and heart muscles, In: M. Markov (Ed.) Electromagnetic Fields in Biology and Medicine, USA, CRC Press, pp. 217-230.

Copyright: (C2016 Arafat M. This is an open-access article distributed under the terms of the Creative Commons Attribution License, which permits unrestricted use, distribution, and reproduction in any medium, provided the original author and source are credited. 